http://jmscr.igmpublication.org/home/ ISSN (e)-2347-176x ISSN (p) 2455-0450 crossref DOI: https://dx.doi.org/10.18535/jmscr/v9i6.49

\title{
Generalized Eruptive Histiocytosis: Mimicking many dermatosis
}

\author{
Authors \\ Dr Sourabh Sharma ${ }^{1}$, Dr GR Tegta ${ }^{2 *}$ \\ ${ }^{1}$ Junior Resident, IGMC, Shimla \\ ${ }^{2}$ Head of Department, Department of Dermatology, Venereology and Leprosy, IGMC Shimla \\ *Corresponding Author \\ Dr GR Tegta
}

\begin{abstract}
Generalized eruptive histiocytosis $(G E H)$ is a rare, benign, self-healing and generalized cutaneous histiocytosis that mainly affects adults and presents with multiple symmetric papules on face, trunk, and proximal extremities. We report a case with atypical presentation of $G E H$, a rare type of non-langerhans cell histiocytosis. GEH is a very close clinico-pathplogical mimicker of not only leprosy and diffuse cutaneous leishmaniasis but a lot of other dermatosis. So a detailed clinical workup, meticulous histopathological examination and most important immmunohistochemical assessment is recommended for early diagnosis. Although spontaneous involution is the rule but a close follow-up is necessary to evaluate for underlying diseases or evolution into more severe types of histiocytoses.
\end{abstract}

Keywords: GEH, Mimicker, Disease.

\section{Introduction}

Generalized eruptive histiocytosis $(\mathrm{GEH})$ is a rare, benign, self-healing and generalized cutaneous histiocytosis that mainly affects adults and presents with multiple symmetric papules on face, trunk, and proximal extremities. ${ }^{1}$ Clinical, histopathological, and immunohistochemical studies play an important role in coming to a final diagnosis and to differentiate it from other infiltrative conditions common in this geographical location, like leprosy. We report a case of a middle-aged woman who presented with generalized asymptomatic papules and nodules and was treated for leprosy but was finally diagnosed to have GEH which had progressed to progressive nodular histiocytosis (PNH).
Mucous membrane lesions are rare and visceral involvement has not been observed, although there are some reports where an association with rheumatic fever, ${ }^{2}$ exanthema subitum $^{3}$ or an underlying neoplasm was noted. ${ }^{4-7}$ Histological examination shows a monomorphous proliferation of benign histiocytes without deposition of lipids, iron or mucine. Electron microscopy reveals that these cells may possess various markers, such as comma shaped bodies, dense bodies and regularly laminated bodies, but no Birbeck granules.

\section{Case Report}

Herein we report a case of GEH in a 41-year-old male labourer of a hilly state with peculiar clinical and immunohistochemical features. Presented with multiple, discrete asymptomatic brown to 
reddish popular and nodular lesions all over the body. Onset of lesions was from face and neck which became symmetrically generalized in a period of one and half month. Denied for visit to area endemic for leishmaniasis. Cutaneous examination revealed enumerable papules, nodules and plaques predominantly over face, neck, trunk and extremities with sparing of scalp, palms, soles and mucosae. No evidence of systemic or neurological involvement on history and clinical examination

Investigation

- Slit skin smear examination from active lesions- No acid fast bacilli or Leishman Donovan (LD) bodies

- Routine hematology, biochemistry, serum angiotensin converting enzyme and lipids profile were normal

- Serological markers for hepatitis, HIV and syphilis were negative

- Chest X-ray, abdominal ultrasonography, CT abdomen and ophthalmological examination revealed no extra-cutaneous involvement

- Initial histopathological examination at a private clinic showed normal to flattened epidermis with sub-epidermal Grenz zone with granulomas in papillary and reticular dermis, with no acid fast bacilli or LD bodies

- A diagnosis of lepromatous leprosy was suggested but because of slit smear negativity and spontaneous resolution of few lesions leaving hyperpigmented macules a review histopathological examination was performed at our institute

- Clinico-pathological correlation generalized eruptive histiocytosis was diagnosed which was confirmed by immunohistochemical positivity for CD68 and negative staining for CD34, S100, CD1a and XIII a factor (Fig. 1)

- No treatment was started as lesions were in resolving phase but a strict follow up was advised

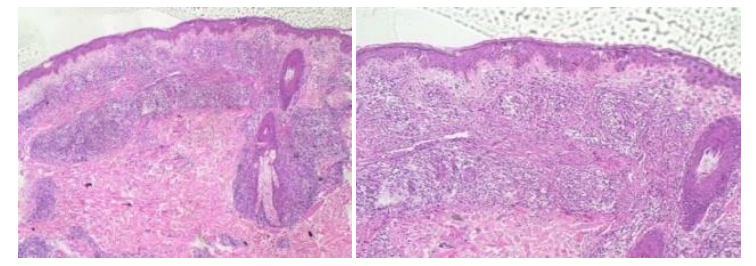

Figure 1. The biopsy shows nodular dense monomorphous granulomatous infiltrate of foamy histiocytes with uniform and sparse scattering of lymphocytes involving the reticular dermis and sparing appendages and upper epidermis. The subepidermal papillary dermis (grenz zone) is spared by the infiltrate. Several histiocytes containing bluish staining foamy material and a few foamy giant cells are also seen in the infiltrate. The macrophage granuloma follows neurovascular bundles in deep reticular dermis.

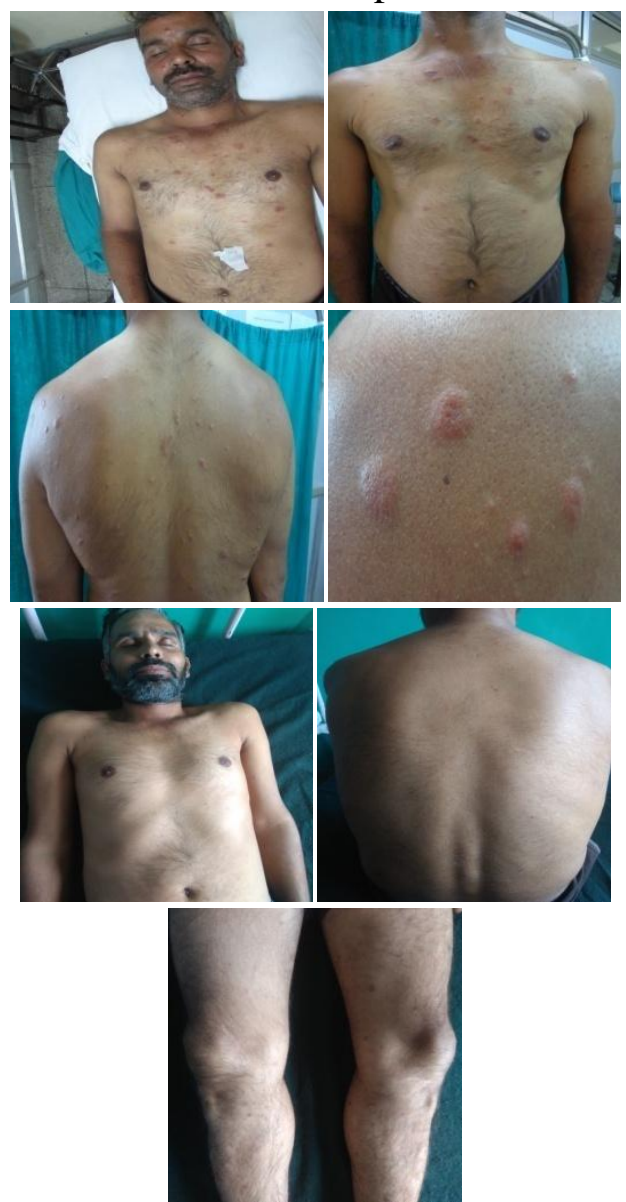

Figure 2. a-d. Papuloplaque lesions over the body upon presentation. e-g: disappeared lesions upon strict follow-up

\section{Discussion}

Histiocytosis is classified into type I - Langerhans cell histiocytosis, type II a - histiocytes involving cells of dermal dendrocyte lineage, type IIb - 
histiocytosis involving cells other than Langerhans cells and dermal dendrocytes, and type III - malignant histiocytic disorders. ${ }^{9}$ GEH is included in Type IIa - reactive histiocytoses involving cells of dermal dendrocyte lineage. GEH is a rare cutaneous histiocytosis that mainly affects adults, ${ }^{9}$ although children with these disorders have been reported. ${ }^{10}$ Ever since Winkelmann and Muller described cases of GEH in three patients in 1963, many cases have been reported. Clinically, GEH presents with multiple asymptomatic skin colored, brownish or blue-red symmetric papules on face, trunk, and proximal extremities which tend to come in crops. Mucosal lesions are rare. Spontaneous resolution of the lesions seen in GEH may be due to apoptosis. GEH may rarely be associated with underlying hematological malignancies like acute monocytic leukemia. ${ }^{11}$

The features in this case which favored the diagnosis of GEH include age of the patient (adult), presence of multiple asymptomatic, skin colored to erythematous, symmetrical papules on face, trunk, and extremities, absence of systemic involvement and absence of mucosal lesions, history of spontaneous resolution of the lesions seen on the extremities, histopathological features of nodular aggregates of large histiocytes and absence of giant cells, and lastly the IHC studies showing histiocytes being CD 68 positive and S 100 negative, and electron microscopy showing absence of Birbeck granules.

The differential diagnosis considered for GEH included lepromatous leprosy (histoid variant); the skin colored infiltrated papules; and plaques predominantly on face involving medial aspect of eyebrows and ear lobe infiltration had led to a diagnosis of Hansen's disease in her native place, the disease being more prevalent in a country like India. Our patient had also received a complete course of MB- MDT for 1 year (WHO guidelines for leprosy treatment) but had only some improvement at times which can be attributed to the spontaneous resolution of lesions seen in GEH. Absence of peripheral nerve involvement clinically and failure to demonstrate AFB in SSS ruled out the possibility of leprosy. Post kalaazar dermal leishmaniasis, sarcoidosis, secondary syphilis, follicular mucinosis, and cutaneous $\mathrm{T}$ cell lymphoma were ruled out based on blood investigations and histopathologic examination. ${ }^{12-}$ 13

\section{Conclusion}

We report a case with atypical presentation of $\mathrm{GEH}$, a rare type of non-langerhans cell histiocytosis. GEH is a very close clinicopathplogical mimicker of not only leprosy and diffuse cutaneous leishmaniasis but a lot of other dermatosis. So a detailed clinical workup, meticulous histopathological examination and most important immmunohistochemical assessment is recommended for early diagnosis. Although spontaneous involution is the rule but a close follow-up is necessary to evaluate for underlying diseases or evolution into more severe types of histiocytoses.

\section{References}

1. Winkelmann RK, Muller SA. Generalized eruptive histiocytoma: a benign popular histiocyticreticulosis. Arch Dermatol 1963; 88: 586-596.

2. Matsushima $\mathrm{Y}$, Ohnishi $\mathrm{K}$, Ishikawa O. Generalized eruptive histiocytoma of childhood associated with rheumatic fever. Eur J Dermatol 1999; 9: 548-550

3. Tamiya H, Tsuruta D, Takeda E, Moriwaki $\mathrm{K}$, Kobayashi H, Ishii M. Generalized eruptive histiocytoma with rapid progression and resolution following exanthema subitum. Clin Exp Dermatol 2005; 30: 300-301.

4. Statham BN, Fairris GM, Cotterill JA. Atypical eruptive histiocytosis - a marker of underlying malignancy? Br J Dermatol 1984; 110: 103-105

5. Saijo S, Hara M, Kuramoto Y, Tagami H. Generalized eruptive histiocytoma: a report of a variant case showing the 
presence of dermal indeterminate cells. J

Cutan Pathol 1991; 18: 134-136.

6. Marzano AV, Facchetti M, Caputo R.

Generalized eruptive histiocytosis

(histiocytoma). Eur J Dermatol 2002; 12:

205-206.

7. Caputo R, Ermacora E, Gelmetti C, Berti

E, Gianni E, Nigro A. Generalized eruptive histiocytoma in children. $\mathrm{J}$ Am Acad Dermatol 1987; 17: 449-454.

8. Ashworth J, Archard L, Woodrow D, Cream JJ. Multiple eruptive histiocytoma cutis in an atopic. Clin Exp Dermatol 1990; 15: 454-456.

9. Repiso T, Roca-Miralles M, Kanitakis J, Castells-Rodellas A. Generalized eruptive histiocytoma evolving into xanthoma disseminatum in a 4-year-old boy. $\mathrm{Br} \mathrm{J}$ Dermatol 1995; 132: 978-982.

10. Zelger BW, Sidoroff A, Orchard G, Cerio R. Non-Langerhans cell histiocytoses. A new unifying concept. Am J Dermatopathol 1996; 18: 490-504.

11. Braun-Falco O, Korting HC, Zienicke H, Klovekorn W. Eruptive histiocytoma and xanthoma disseminatum as manifestations of the same disease? Hautarzt 1988; 39: 652-657.

12. Sidoroff A, Zelger B, Steiner H, Smith N. Indeterminate cell histiocytosis: a clinicopathological entity with features of both $\mathrm{X}$ - and non-X histiocytosis. $\mathrm{Br} \mathrm{J}$ Dermatol 1996; 134: 525-532.

13. Ferrando J, Campo-Voegeli A, SolerCarrillo J, Munoz E, Sole M, Palou J. Systemic xanthohistiocytoma: a variant of xanthoma disseminatum? Br J Dermatol 1998; 138: 155-160. 